Somnologie 2013 - 17:5-5

DOI 10.1007/s11818-013-0605-1

Online publiziert: 6. März 2013

(c) Springer-Verlag Berlin Heidelberg 2013

\section{Geert Mayer}

Hephata Hessisches Diakoniezentrum e. V., Schwalmstadt

\title{
Offene Fragen der Schlafmedizin
}

Haben wir uns in der Schlafmedizin immer die richtigen Fragen gestellt, die richtigen Methoden angewendet, um die Schlafmedizin weiterentwickeln zu können? Jeder Schlafmediziner, der sich intensiv mit einem Teilgebiet seines großen interdisziplinären Fachs beschäftigt, wird sich diese Fragen stellen.

Spätestens nach fast 50 Jahren mit den Regeln von Rechtschaffen u. Kales und der Veröffentlichung der neuen und neuesten American-Academy-of-Sleep-Medicine (AASM)-Kriterien müssen wir feststellen, dass unsere Methoden limitiert sind und neue Regeln nicht unbedingt zu neuen Erkenntnissen führen oder die Methoden an sich verbessern.

Unsere Methoden beziehen sich längst nicht mehr nur auf die Erfassung der Schlafmikro- und -makrostruktur, sondern auf die Zusammenhänge von Schlaf und Gedächtnis, Metabolismus, Genetik etc. Oft werden wir mit erstaunlichen neuen Erkenntnissen konfrontiert, die bei genauer Betrachtung und Verständnis der Methodik zeigen, dass sie auf bestimmten mathematischen oder statistischen Prämissen beruhen, die, wenn sie modifiziert werden, zu ganz anderen Ergebnissen führen. Häufig sind diese Ergebnisse nicht reproduzierbar und erfüllen damit nicht die Kriterien von Wissenschaftlichkeit. Dennoch können sie neue Fragen aufwerfen, die der Beantwortung der initialen Frage dienen können. Denken Sie beispielsweise an die umwerfenden Ergebnisse aus genetischen genomweiten Studien. Sie zeigen Assoziationen mit bestimmten Genen, die aber wiederum nur einen kleinen Anteil der Krankheitsursache(n) erklären. Gleiches kann auch über die bildgebenden Verfahren gesagt werden. Statistische Korrekturverfahren kön- nen zum Teil anfängliche signifikante $\mathrm{Zu}$ sammenhänge bedeutungslos werden lassen. Auch die Interpretation der Befunde birgt methodische Schwierigkeiten und kann systemimmanent bleiben oder sich in Spekulationen verlieren.

Die Autoren dieses Schwerpunkthefts "Offene Fragen der Schlafmedizin“ beleuchten die Methoden und Paradigmen ihrer Forschung in kritischer Weise. Sie werfen Fragen auf, die den Weg für neue Untersuchungsansätze eröffnen.

Lassen Sie sich also von den Möglichkeiten inspirieren, bisheriges Wissen zu hinterfragen, die gewohnte Methodik zu verändern und zu lernen, die richtigen Fragen zu stellen. Dies wird dazu beitragen, die Schlafmedizin als ernst zu nehmende Wissenschaft weiter zu etablieren. Erst wenn es uns gelingt, für Konzepte wie Vigilanz, Schläfrigkeit, Müdigkeit etc. quantitative und reproduzierbare Messmethoden zu entwickeln und zu standardisieren, kommen wir einen Schritt weiter.

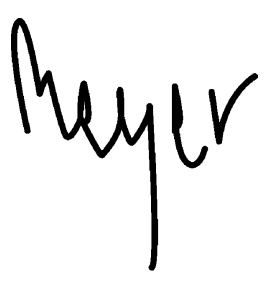

G. Mayer

\section{Korrespondenzadresse}

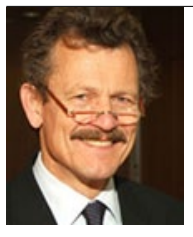

Geert Mayer

Hephata Hessisches Diakoniezentrum e. V. Schimmelpfengstr. 6, 34613 Schwalmstadt geert.mayer@hephata.com 